\title{
A decade of malaria during pregnancy in Brazil: what has been done concerning prevention and management
}

\author{
Paola Marchesini', Fabio Trindade Maranhão Costa ${ }^{2}$, Claudio Romero Farias Marinho'/+ \\ 'Departamento de Parasitologia, Instituto de Ciências Biomédicas, Universidade de São Paulo, São Paulo, SP, Brasil \\ ${ }^{2}$ Departamento de Genética, Evolução e Bioagentes, Universidade Estadual de Campinas, Campinas, SP, Brasil
}

\begin{abstract}
In Brazil, malaria remains a disease of major epidemiological importance because of the high number of cases in the Amazonian Region. Plasmodium spp infections during pregnancy are a significant public health problem with substantial risks for the pregnant woman, the foetus and the newborn child. In Brazil, the control of malaria during pregnancy is primarily achieved by prompt and effective treatment of the acute episodes. Thus, to assure rapid diagnosis and treatment for pregnant women with malaria, one of the recommended strategy for low transmission areas by World Health Organization and as part of a strategy by the Ministry of Health, the National Malaria Control Program has focused on integrative measures with woman and reproductive health. Here, we discuss the approach for the prevention and management of malaria during pregnancy in Brazil over the last 10 years (2003-2012) using morbidity data from Malaria Health Information System. Improving the efficiency and quality of healthcare and education and the consolidation of prevention programmes will be challenges in the control of malaria during pregnancy in the next decade.
\end{abstract}

Key words: malaria - pregnancy - epidemiology - control - Brazil

Approximately 125 million pregnant women worldwide are exposed to the risks of malaria in pregnancy (MiP) each year, resulting in 200,000 infant deaths (Steketee et al. 2001, Dellicour et al. 2010). In malaria hyper and holoendemic regions, pregnant women together with children less than five years old are the most susceptible individuals to Plasmodium spp infections. The symptoms and complications of MiP vary according to the transmission intensity and level of acquired immunity. Overall, MiP can result in stillbirth, spontaneous abortion, low birth weight (birth weight $<2.5 \mathrm{~kg}$ ) and neonatal and maternal death. Pregnant women have an overall decrease in resistance to infections and, when living in areas of low or unstable malaria transmission, such as some areas of Brazilian Amazon may have little or no immunity to malaria. They present a two-threefold higher risk of developing severe effects compared to their non-pregnant peers. Interestingly, the disease severity is normally increased in first and second pregnancies, suggesting that robust protective immunological mechanisms are induced after several pregnancies (Brabin et al. 2004). In addition, there appears to be an increased susceptibility to Plasmodium falciparum in-

\footnotetext{
doi: 10.1590/0074-0276140047

Financial support: FAPESP, CNPq, CAPES, INCTV (CNPqFAPEMIG)

PM was sponsored by CAPES-PNPD fellowships and CRFM and FTMC are CNPq fellows.

+ Corresponding author: marinho@usp.br

Received 7 February 2014

Accepted 2 May 2014
}

fection or disease even in areas where malaria by other plasmodial species are more prevalent (Martínez-Espinosa et al. 2004, Parekh et al. 2007).

In Brazil, malaria transmission occurs almost exclusively in the Amazon Region (99.6\% of cases), where Plasmodium vivax and P. falciparum coexist. P. vivax is the most important malaria species associated with infection, since it accounts for around $85 \%$ of the registered cases, contrasting with the situation in most of Africa and in Asia, where MiP is typically caused by $P$. falciparum. In the last decade, Brazil has successfully reduced both malaria morbidity and mortality. The National Malaria Control Program (NMCP) is responsible for funding disease control measures and formulates the major policies that are followed by all of the states and municipalities. However, funding is managed in a decentralised manner. The total number of malaria cases reported in Brazil has dropped almost two-fold, from 635,646 cases in 1999 to approximately 235,000 cases in 2012 (Figure). Data subject to revision showed approximately 178,000 cases in 2013 . This significant reduction was observed in all groups at risk, including pregnant women. Nevertheless, in Brazil, the pathogenic mechanisms involved in MiP are still puzzling, which makes its real burden unknown and indicates the urgency of intense investigation (Costa et al. 2012).

In 2006, the NMCP published a technical note with some recommendations for the treatment and care of pregnant women in endemic areas. According to it, all women undergoing prenatal care should be tested for malaria at every antenatal care (ANC) visit to ensure faster diagnosis and treatment and it was emphasised that healthcare professionals should ensure compliance with treatment. Moreover, as the use of insecticide- 

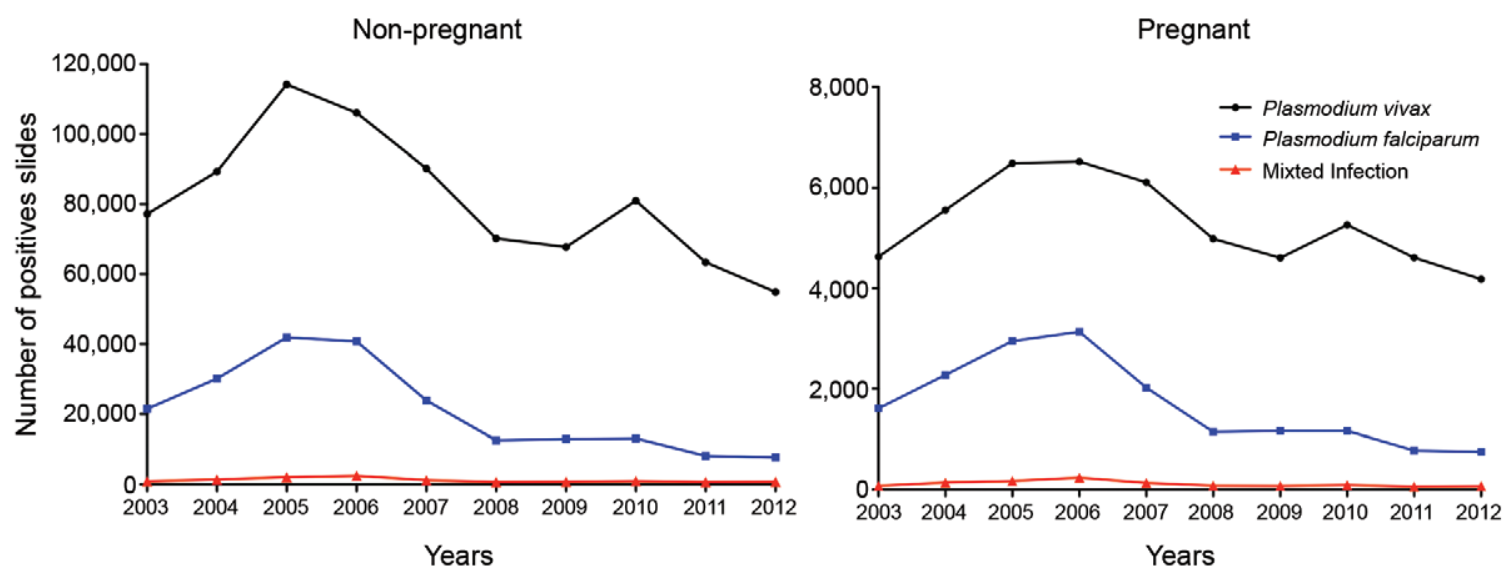

Number of positive slides by species, non-pregnant and pregnant women. Source: Epidemiological Surveillance Information System-Malaria.

treated nets (ITNs) demonstrated a significant impact on decreasing the exposure to infective mosquito bites (Gutman \& Kachur 2010), the NMCP began the free distribution of ITNs to municipalities with a greater malaria burden in 2010. The rationale was to ensure coverage of at least $80 \%$ of these areas, thereby reducing transmission and improving individual protection for the entire population of these areas, including pregnant women and children less than five years of age.

Another strong element of the malaria prevention and control strategy is based on a robust, locally available and up-to-date information system, allowing the identification of essential epidemiological variables. In Brazil, the Epidemiological Surveillance Information System (SIVEP)-Malaria is used for the notification of every malaria case diagnosed (de Almeida et al. 2010). Consequently, every diagnosed case is properly notified and patients are treated promptly.

Pregnant women must receive prompt treatment with effective antimalarial medication accompanied by the recommended supportive care. Close collaboration between malaria control and reproductive health programmes is needed to facilitate the development of systematic management protocols and drug supply strategies. In 2014, the so-called "Stork Network" will reinforce NMCP recommendations and emphasise that the malaria slide test in endemic region should be performed during the established standard six or more prenatal visits. In fact, the number of consultations during prenatal care is directly related to the best indicators of maternal and child health. However, executing this strategy will be a challenging task due to the difficulty of implementing a system capable of assuring an adequate number of visits.

Observational data showed that the proportion of live births with seven or more prenatal consultations increased from $46.2 \%$ in 2000 to $58.8 \%$ in 2009 . In gener$\mathrm{al}$, there have been improvements in healthcare for pregnant women, but it should be noted that this indicator hides important inter-regional differences in Brazil. For instance, in 2010 in the southern region of Brazil, 75.3\% of live births followed at least seven prenatal visits, whereas this proportion dropped to $36.8 \%$ for the states in the northern region. Moreover, differences were also observed regarding states from the same region. For example, in the northern region, this proportion was $49 \%$ in the state of Rondônia, in contrast to $31.1 \%$ in the state of Acre (dssbr.org/site/). These observations clearly indicate profound regional discrepancies.

Studies of MiP have been more frequent and are supported by the Ministry of Health in Brazil because awareness of the deleterious effects of this disease on the mother and foetus has been placed on the agenda of countries of low and moderate endemicity. Preventive programmes delivered through $\mathrm{ANC}$ frequently face challenges due to late ANC attendance and non-compliance, which can limit the impact of interventions during pregnancy and give rise to a null result. Therefore, at the local level in Brazil, there is a need to integrate malaria control and reproductive health programmes to facilitate malaria diagnosis and treatment for pregnant women, preferably at the same site. The Family Health Program should monitor compliance to treatment, for example, through daily visits by healthcare workers. The use of ITNs should be monitored to ensure that pregnant women use bed nets throughout the gestational period. Healthcare workers should be trained to provide these recommendations to pregnant women during their ANC visits and during home visits of Family Health Program healthcare workers (Ndyomugyenyi et al. 2011). In addition to monitoring quinine efficacy, it is very important to pay special attention to the pharmacovigilance of antimalarial drugs used during pregnancy, aiming for improvements in treatment for $P$. falciparum during the first trimester.

In conclusion, although pregnant and non-pregnant women present the same patterns regarding the number of cases according to SIVEP, the risks of disease severity are still a threat for pregnant women with malaria. Thus, the NMCP needs to take special measures to protect pregnant women from malaria infection and from the deleterious effects to the mother and her baby, taking into consideration their specific and local circumstances. We lastly believe that community-based awareness and educational programmes are essential to educate women about the risks associated with malaria during pregnancy. 


\section{REFERENCES}

Brabin BJ, Romagosa C, Abdelgalil S, Menendez C, Verhoeff FH, McGready R, Fletcher KA, Owens S, D'Alessandro U, Nosten F, Fischer PR, Ordi J 2004. The sick placenta-the role of malaria. Placenta 25: 359-378.

Costa FTM, Lopes SCP, Albrecht L, Ataíde R, Siqueira AM, Souza RM, Russell B, Renia L, Marinho CRF, Lacerda MVG 2012. On the pathogenesis of Plasmodium vivax malaria: perspectives from the Brazilian field. Int J Parasitol 42: 1099-1105.

de Almeida LB, Barbosa MGV, Martínez-Espinosa FE 2010. Malária em mulheres de idade de 10 a 49 anos, segundo o SIVEPMalária, Manaus, Amazonas, 2003-2006. Rev Soc Bras Med Trop 43: 304-308.

Dellicour S, Tatem AJ, Guerra CA, Snow RW, ter Kuile FO 2010. Quantifying the number of pregnancies at risk of malaria in 2007: a demographic study. PLoS Med 7: 1-10.

Gutman J, Kachur SP 2010. Treating malaria in pregnant women: a pressing problem. Lancet Infect Dis 10: 739-740.
Martínez-Espinosa FE, Daniel-Ribeiro CT, Alecrim WD 2004. Malaria during pregnancy in a reference centre from the Brazilian Amazon: unexpected increase in the frequency of Plasmodium falciparum infections. Mem Inst Oswaldo Cruz 99: 19-21.

Ndyomugyenyi R, Clarke SE, Hutchison CL, Hansen KS, Magnussen P 2011. Efficacy of malaria prevention during pregnancy in an area of low and unstable transmission: an individually-randomised placebo-controlled trial using intermittent preventive treatment and insecticide-treated nets in the Kabale Highlands, southwestern Uga. Trans R Soc Trop Med Hyg 105: 607-616.

Parekh FK, Hernandez JN, Krogstad DJ, Casapia WM, Branch OH 2007. Prevalence and risk of Plasmodium falciparum and $P$. vivax malaria among pregnant women living in the hypoendemic communities of the Peruvian Amazon. Am J Trop Med Hyg 77: 451-457.

Steketee RW, Nahlen BL, Parise ME, Menendez C 2001. The burden of malaria in pregnancy in malaria-endemic areas. Am $J$ Trop Med Hyg 64 (Suppl. 1-2): 28-35. 